\title{
Creation of Dynamic Virtual Organizations for Child Abuse Prevention in the VO Breeding Environment Context
}

\author{
Pedro Sanz Angulo* and Juan José de Benito Martín \\ University of Valladolid, Industrial Engineering School. \\ Paseo del Cauce, 59. 47011 Valladolid, Spain \\ \{psangulo, debenito\} @eis.uva.es
}

\begin{abstract}
Child abuse is a serious problem in our society. In order to provide an adequate response, all the entities involved in its prevention, detection and intervention should work in collaborative networks. In this sense, our labor aims at facilitating the creation of DVOs adapted to the different abuse situations. To that end, we are working to define both a VBE in the Castilla y Leon region (Spain) and a multi-agent expert system able to facilitate the selection of partners.
\end{abstract}

Keywords: Child Abuse Prevention, Dynamic Virtual Organization, VO Breeding Environments, Partner Selection, Multi-Agent and Expert Systems.

\section{Introduction}

Child abuse is a universal phenomenon that our society must try to completely eradicate as soon as possible. This goal, undoubtedly ambitious, requires an intensive work in the education of every individual and, of course, in the recognition of the children's rights.

Although in recent years significant progresses have been made in this sense, our efforts must continue in order to protect children, i.e., to respond promptly and in a personalized way to each abuse situation. For this purpose, we must employ all the available resources and search for new solutions and tools.

Furthermore, all stakeholders (both entities and people) in the prevention and solution of child abuse must work together creating collaborative networks and providing to the group what they do best, i.e., their core business.

This need leads us to consider the formation of Dynamic Virtual Organizations (DVO) tailored to each particular abuse situation. However, the efficient creation of DVOs only is possible within the VO Breeding Environment (VBE) context.

Based on these ideas, our current work is focused both on the definition of a VBE for our region (Castilla y Leon - Spain) and on the development of a multi-agent expert software tool able to support the selection process in an efficient way.

* This work is derived from the participation of the authors in a research project financed by the Castilla y Leon region (Spain), with reference number VA009A09, entitled "Information and Communication Technologies in the Creation of Organizational Networks: Application in the Field of Child Abuse Prevention." 
The introduction of the VBE concept in conjunction with the use of software agents whose behavior is guided by an expert system is a novelty in the field of child abuse prevention. This combination will allow us optimizing the use of resources and facilitating communication between institutions and professionals, making possible a more agile response of the DVO created ad hoc for the abuse problem.

This document aims, first, at justifying the use of DVO-VBE concepts for the child abuse problem and, second, at presenting the tool we are developing for the selection of partners in these sceneries, the VCAP platform.

\section{The Context: Child Abuse Problem and Networking}

The United Nations Convention on the Rights of the Child 1989 defines child abuse as [1] "all forms of physical or mental violence, injury or abuse, neglect or negligent treatment, maltreatment or exploitation, including sexual abuse, while in the care of parent(s), legal guardian(s) or any other person who has the care of the child." In short, we can say that there is child abuse if children's rights are not respected and there is not a response to their needs [2]; we cannot forget that abuse is, primarily, the lack of good treatment.

There is a great variety of abuse types according to its forms (physical, sexual, psychological, neglect, etc.), the place, the involved actors, the degree of intensity, and so on. Each abuse situation is unique and requires, of course, a different approach: an appropriate response must be designed to each particular case. In this sense, prevention is the best possible approximation to the problem.

Prevention may take place at three different levels: a) primary prevention aims to decrease the incidence or onset of abuse cases, through child advocacy and special attention to their needs, b) secondary prevention is addressed to social groups, families or individuals classified as "high risk" to avoid some situations that end up in abuse, and finally, c) tertiary prevention tries to reduce the duration and severity of the consequences of the problem (stage of rehabilitation or cure). As we will see, our work can be perfectly included in the latter two levels of prevention, and especially in the tertiary one.

Networking is, clearly, the best way for creating a collaborative workspace where to achieve the pursued goals. It is based on the communication of the agents and institutions, so an optimum exchange of information, both qualitative and quantitative, must exist between them.

The originators of the sociotherapeutic networking were Speck and Attneave [3]. They created therapeutic teams to intervene in families in crisis, in the United States, in order to break destructive patterns of family relationships and provide support for alternative options. Several years later, in the eighties, Elkaim [4] carried out the first practice at European level in deprived areas of Belgium. Since then, the networking experiences have evolved and multiplied.

In our country, Spain, we find the networking pilot program of Burlada (Pamplona). Its promoters recognize that one of the challenges of any network program is to provide a process able to organize the different institutional levels and professional resources, in order to ensure the creativity and competence of each one of these instances [5]. 
These and other works, experiences and projects emphasize the need of networking as a means to achieve the ultimate goal of prevention and resolution of child abuse, establishing guidelines and procedures that normally are constructed ad hoc. However, they do not possess software tools capable of solving the different problems of such relationships, e.g. the need for a rapid intervention.

\section{Justification of Our Work}

Network collaboration must face several challenges to become a truly effective instrument to combat the child abuse problem. Every abuse case is unique, so networks must involve different components in each new situation, and even these components can vary during the performance. It is necessary, therefore, a dynamic and flexible networking model that allows the continuing evolution of resources and services, as well as the incorporation of new ones.

These inter-collaborative problems in child abuse domain are difficult to solve without considering the new organizational models and the Information and Communication Technologies (ICT) that make them possible. In this way, the DVOs are, probably, the best response to the problem.

In a DVO a set of business partners come together dynamically, on demand, and in accordance with the requirements and needs [6] of the problem, disappearing when those needs have been met. DVOs are rapid creation and fast dissolution organizations [7], constructed ad hoc the opportunity of collaboration.

However, the effective creation of a DVO only is possible in the context of what Afsarmanesh and Camarinha-Matos [8] have called Virtual Organization Breeding Environments. The VBEs are clubs of organizations prepared to work in long term relations and from which temporary coalitions emerge able to respond dynamically to the different business opportunities [9].

Obviously, there are several similarities between networking that a child abuse situation requires and the networking of the DVOs. We can start, for example, talking about the VBE. In the child abuse domain it is obvious that the VBE would be constituted by any entity or person involved in the solution: schools, social services, health centers, youth services, law enforcement agencies, state security bodies, and so on. All of them bring to the partnership their core business, what they do best, so the VBE contains all necessary processes that the abuse situation requires. In addition, these entities are required to share certain culture of work, ICT infrastructure, etc., to achieve the highest possible performance.

After identifying a new case of abuse (either in the school, or in a health center, or through a complaint at the police station or in the child line, etc.), it is time to go to the VBE and select the set of institutions and/or persons that are the best prepared to respond to the particular problem.

The union of the selected entities configures, ultimately, the DVO responsible for providing the personalized answer to the child abuse situation. Naturally, different DVOs can arise from the breeding environment to provide this response, although the selection of the best one depends both on the requirements and needs of the particular situation of abuse and on the features and capacities of the partners. 
After the selection process, partners need to work together and coordinate their actions in order to find an efficient and agile solution to the problem. This operation phase lasts until an adequate response to the initial problem has been provided. That moment coincides, precisely, with the dissolution of the DVO.

In addition, whenever it is necessary, more partners can be searched, or even their number can be reduced, according to the changes of each abuse case. In short, during the operation of the DVO a reconfiguration phase can take place.

Finally, we must also notice that the entities of a particular DVO can be partners of another consortium which tries to response to a different abuse case.

\section{The VCAP Multi-agent Expert Platform}

Our fundamental objective is to enhance and develop a networking model through the creation of DVOs capable of facing child abuse problems in an agile and efficient way. For this purpose, it is necessary to align the VBE and DVO concepts to the child abuse domain and provide efficient tools to support its formation and operation.

In this paper we propose a multi-agent approach to support the selection process in this context. This technology provides several advantages over other proposed methods, especially for its ability to manage complex and distributed problems. But in addition, our agents have an intelligent behavior through a rule-based Expert System (ES) implemented in their decision module.

Through this platform the best combination of partners is defined dynamically, in real time, based on the system state, the predefined objectives and the features both of the problem and of the stakeholders. The identification and assignment of partners emerge from the interaction between agents, through different negotiation mechanisms. In this sense, we can introduce the Virtual Child Abuse Prevention (VCAP) concept.

\subsection{Analysis of the VCAP Multi-agent Expert System}

The analysis of the platform has been made following the steps defined by Nikraz, Caire and Bahri [10]. Thus, we have identified the different types of agents and users in the system, along with their responsibilities and the acquaintance relationships that exist between them. The result is the agent diagram shown in Fig. 1.

Taking into account the agent container architecture of JADE (Java Agent Development Environment), we have defined the distributed structure of the Fig. 2. As seen in this figure, there is a main container on the manager server in which the VCAP Management Agent resides, in addition to the Information Provider Agent, the Yellow Pages Agent (where the Resource Agents register their services) and the rest of agents responsible for the management of the Jade platform.

Also there are multiple remote containers: specifically, so many remote containers as institutions and professionals the breeding environment has. In each of these containers we can identify the VBE Member Agent (associated to the different actors involved in the solution) and an Access Agent for security issues. 


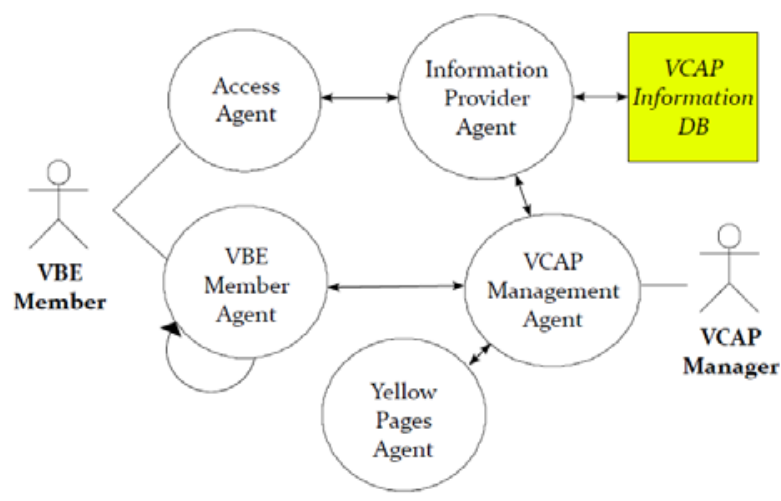

Fig. 1. Agent diagram for the VCAP platform

We have considered that all of these entities can provide their core business and, at the same time, be the promoters of the DVO formation process. It is also possible that a third entity or person starts the process, but for now we just consider that the system is open only to the VBE members. Perhaps in the future the platform can be open to everyone, but previously it is necessary to solve different security problems.

The union of these remote containers with the main container results into the distributed agent platform shown in the Fig. 2.

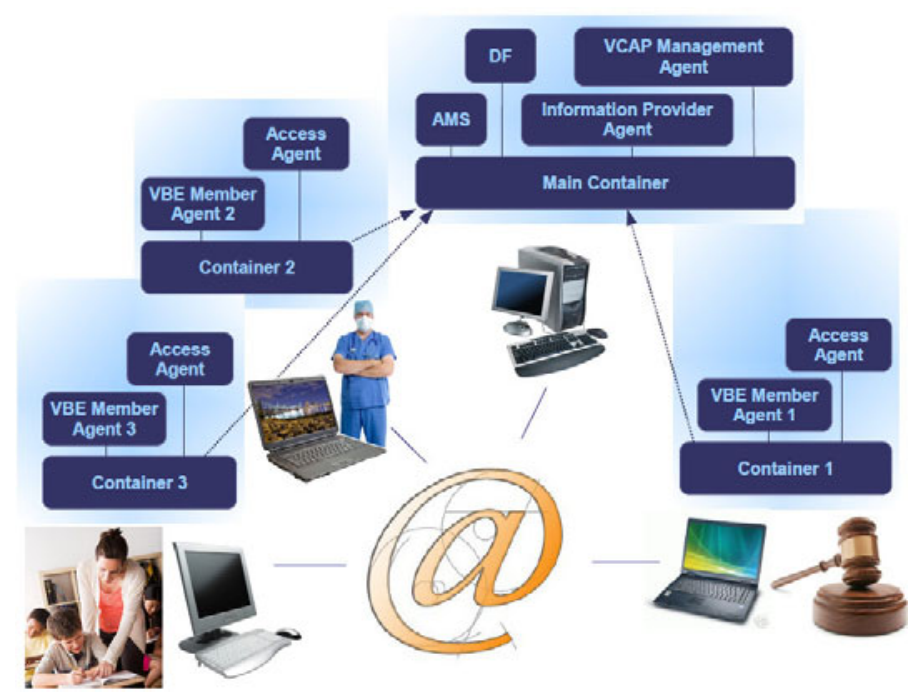

Fig. 2. Distributed structure of the VCAP platform

\subsection{Current Development of the VCAP Platform}

The current development of the VCAP platform is possible thanks to our experience in the creation of the DVEBreeder tool (see Fig. 3), a multi-agent expert platform developed to support the selection of partners in the enterprise domain [11]. 


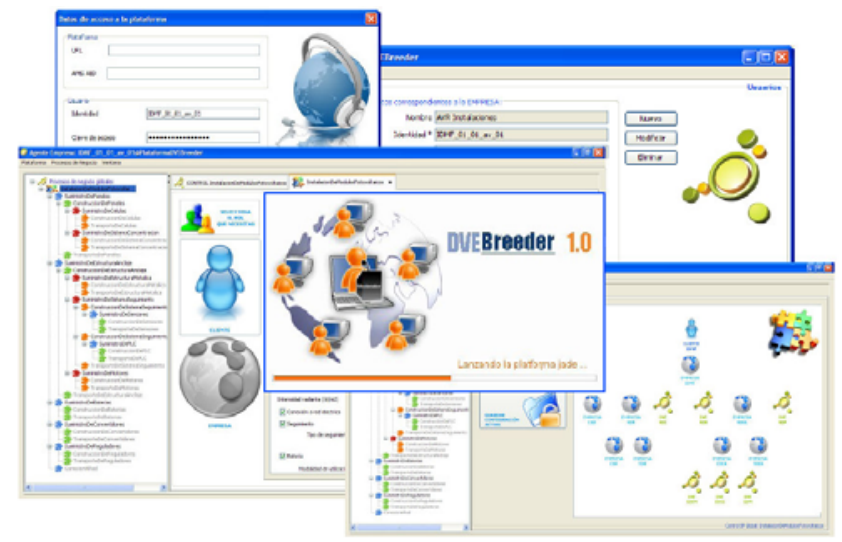

Fig. 3. Some snapshots of the DVEBreeder platform

The VCAP platform inherits many of the features of its predecessor: the integration of multi-agent and expert system technologies; the identification of the intelligent agents is made through an multi-approach model that takes into account the VBE concept; the negotiation between autonomous agents is based on the knowledge embodied in its decision modules; the communication between agents is quite simple thanks to the platform used in the MAS development (Jade); the behavior of agents is quite similar to the behavior that a human expert can have thanks to JESS, the rulebased expert system; it is flexible, dynamic and scalable; FIPA standards are met; the interface is intuitive and the code reuse is simple thanks to its open structure, etc.

In summary, VCAP is an innovative tool able to solve the CAP problem in an agile and efficient way. Fig. 4 shows a simplified scheme of the platform performance in the application domain.

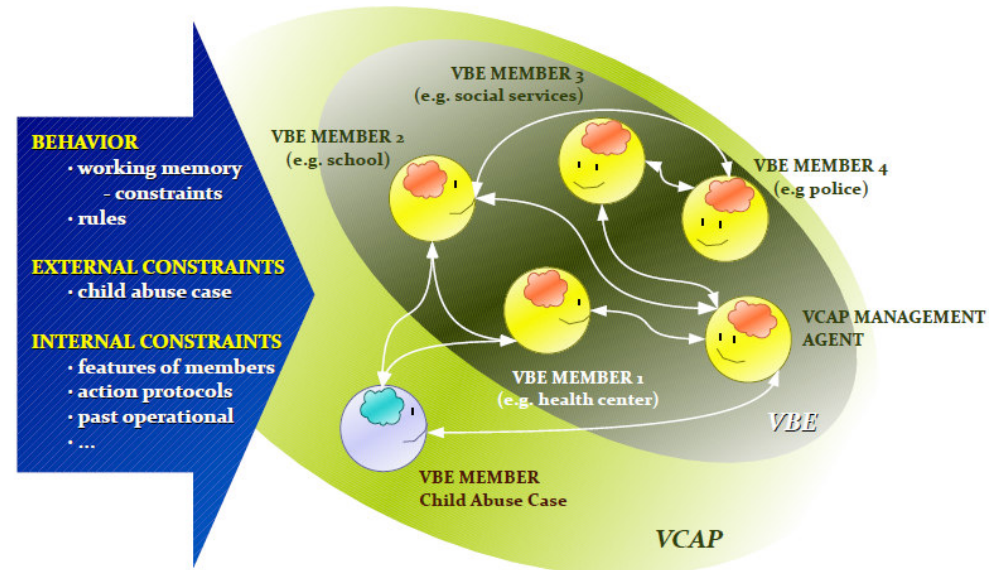

Fig. 4. Simplified scheme of the VCAP platform performance 


\section{Conclusions}

In this article we have tried to justify the use of DVO and VBE concepts in the child abuse domain. In this sense, we also have presented a multi-agent expert solution in which DVOs emerge from the VBE context. After having done the planning and analysis, we are currently working in the design and implementation phases. To do this, we need to define some elements of the platform as e.g. the application domain ontology, service descriptions, agent decision modules, etc. Others, such as the agent interactions, message templates, interaction protocols or the ontologies for the management of the platform, are very similar to those established in the DVEBreeder tool, what will greatly facilitate the final implementation of the platform.

Acknowledgments. We would like to express our gratitude to the managers and members of REA, the Association for the Children and Youth Defense of Castilla y León, and, in particular, to Ms $\mathrm{M}^{\mathrm{a}}$ Elena Villa Ceinos.

\section{References}

1. United Nations - Office of the High Commissioner of Human Rights. Convention on the Rights of the Child, resolution 44/25 of November 20 (1989), http: / / www2 . ohchr. org/english/law/crc. htm (Last visit in February 2010)

2. Sanz, P., Villa, M.E., Manso, M.A., De Benito, J.J.: Aplicación de la Tecnología Multiagente y los Sistemas Expertos al trabajo en Red para la Prevención del Maltrato Infantil. In: IX Congreso Estatal de Infancia Maltratada, Valladolid, Spain (2008)

3. Speck, R., Attneave, C.: Redes Familiares. Amorrortu Editores, Buenos Aires (1974)

4. Elkaïm, M.: Las prácticas de la terapia de red. Gedisa, Barcelona (1989)

5. De Miguel, M., Fernández, M.: Detección precoz del maltrato infantil. Programa piloto de trabajo en red. Anales del Sistema Sanitario de Navarra 25(2), 25-34 (2002)

6. Ouzounis, E.K.: An Agent-Based Platform for the Management of Dynamic Virtual Enterprises. PhD thesis. University of Berlin (2001)

7. Browne, J., Zhang, J.: Extended and Virtual Enterprises - Similarities and Differences. International Journal of Agile Management Systems 1(1), 30-36 (1999)

8. Afsarmanesh, H., Camarinha-Matos, L.M.: A Framework for Management of Virtual Organization Breeding Environments. In: Collaborative Networks and their Breeding Environments, pp. 35-48. Springer, Boston (2005)

9. Camarinha-Matos, L.M., Afsarmanesh, H., Ollus, M.: ECOLEAD: a Holistic Approach to Creation and Management of Dynamic Virtual Organizations. In: Collaborative Networks and their Breeding Environments, pp. 3-16. Springer, Boston (2005)

10. Nikraz, M., Caire, G., Bahri, P.A.: A Methodology for the Analysis and Design of MultiAgent Systems Using JADE. Int. J. of Computer Systems Science and Engineering (2006)

11. Sanz, P., De Benito, J.J.: Design and Implementation of a Multi-agent Framework for the Selection of Partners in Dynamic VEs. In: Leveraging Knowledge for Innovation in Collaborative Networks, pp. 341-348. Springer, Heidelberg (2009) 